\title{
Fetal heart rate baseline estimation with analysis of fetal movement signal
}

\author{
Yaosheng Lu ${ }^{\mathrm{a}}{ }^{*}$, Xiaodong $\mathrm{Li}^{\mathrm{a}}$, Shouyi $\mathrm{Wei}^{\mathrm{b}}$ and Xiaolei Liu ${ }^{\mathrm{c}}$ \\ ${ }^{a}$ Department of Electronic Engineering, Jinan University, Guangzhou 510632, China \\ ${ }^{b}$ Department of Biomedical Engineering, Washington University in St. Louis, St. Louis, USA \\ ${ }^{c}$ Department of Electrical and Electronic Engineering, University of Hong Kong, Hong Kong, China
}

\begin{abstract}
In order to assign appropriate baseline estimation algorithms to different fetal heart rate tracing, a method to evaluate the fetal heart rate (FHR) baseline combining with the fetal movement information was proposed. Fetal actography and tocography were used to extract the fetal movement information. The results showed that the combined method, where the fetal movement detection result was the union of results of actography and tocography, achieved a better performance with the highest sensitivity and an acceptable positive predictive value (PPV). Furthermore, the mean absolute errors (MAEs) of basal FHR values between the two algorithms and the expert were calculated with respect to the duration coefficient of fetal movement. The results showed that the algorithm using empirical mode decomposition (EMD) and Kohonen neural network $(\mathrm{KNN})$ had lower MAEs than a traditional linear baseline estimation algorithm as the duration coefficient increased. However, if the duration coefficient is below 0.2, the errors may be tolerant for the FHR baseline estimation by a linear baseline estimation algorithm, which indicates that different algorithms may be selected for FHR baseline estimation based on different duration coefficients of fetal movement.
\end{abstract}

Keywords: Fetal heart rate, baseline estimation, fetal movement, Ostu method

\section{Introduction}

Fetal heart rate (FHR) baseline estimation plays a critical and essential role in the analysis for cardiotocography (CTG), therefore, a number of FHR baseline estimation algorithms have been proposed in recent decades [1-8]. The existing baseline estimation algorithms mainly adopt linear methods like low-pass filters or time-domain average method, which may not adapt to the complex or irregular signals obtained in antepartum fetal heart monitoring. Therefore, a non-linear FHR signal baseline estimation algorithm using empirical mode decomposition (EMD) and computational intelligence tool of Kohonen neural network (KNN) was proposed previously and proven to have a satisfactory effect, especially for the FHR signals containing long-term and frequent accelerations [9]. However, the EMD-KNN method suffers predominantly from low computational efficiency. As a result, the exact discrimination between regular and irregular signals with long-term frequent accelerations is considered helpful to determine which baseline estimation algorithm is employed.

${ }^{*}$ Corresponding author: Yaosheng Lu, Department of Electronic Engineering, Jinan University, Guangzhou 510632, China. Tel.: +86 20 85220231; Fax: +86 20 87583004; E-mail: tluys@jnu.edu.cn. 
As the FHR accelerations have been demonstrated with a temporal correlation with fetal movement, which means that the more and larger accelerations are, the more and longer-duration fetal movement will be [10-12], fetal movement can be used to differentiate the types of FHR signals.

There are several measures to record fetal movement [13]. Doppler fetal actograph has been developed as an effective method with a band-pass filter that filters the demodulated Doppler shift signal from the ultrasound probe, which is believed to be the frequency band containing the information of fetal movement [14]. TOCO signal (or tocography) is also a source of fetal movement, which contains some information of fetal movement inevitably during acquisition, despite the $56 \%$ of the true positive rate of the tocography that has been clinically proved in [15]. More traditionally, the patient or doctor can use an event marker to record fetal movement whenever she feels the fetus' movement.

In this study, the approaches to extract fetal movement signals from actography and tocography were presented. Then a combinational baseline estimation method consists of the EMD-KNN method and a traditional baseline estimation method described in [8] was presented, considering the duration of fetal movement.

\section{Materials and methods}

\subsection{Acquisition and preprocessing}

Fifty-two experimental signals, including antenatal FHR, TOCO and fetal movement tracing, were clinically recorded from the People's Hospital of Baiyun District, Guangzhou. All the tracing were measured simultaneously with a period of 20 minutes. During monitoring, an experienced obstetric expert with more than 30 years of experience recorded the fetal movement artificially by pressing the abdomen of the pregnant women.

Due to the data acquisition problem, there are a number of missing points or artifacts in the original FHR tracing. In order to recover the undisturbed FHR signals, these artifacts are detected and removed, and linear interpolation is used to fill the gaps.

As to fetal movement tracing, a band-pass filter is used to reject the background noises further. Then a de-noising algorithm described in [16] is used to de-noise the fetal movement signals.

\subsection{Baseline estimation of the FHR signals}

The predominant task of this study is to find out a better method for the baseline estimation of the FHR signals, and two methods are selected based on our previous research [9]: an improved method from Mantel's [8] (marked as Alg1) and the EMD-KNN algorithm (marked as Alg2).

Alg1 adopts a criterion to select a peak which is obtained by histogram analysis of the entire FHR signal. Then a smoothing filter with forward and backward propagation is used to obtain a pliable baseline that does not follow accelerations or decelerations entirely. Replacement of segments in the FHR signal with the resulted baseline is done after performing the filtering. The same process is repeated four times to achieve the final FHR baseline.

Alg2 adopts EMD method to reconstruct the FHR signal so as to remove noise while retaining the typical FHR changes. For the reconstructed signal, the local extreme points are found to form the training set which is input into KNN to conduct cluster analysis. The characteristic points of the baseline part are confirmed as the output result of KNN. On the basis of these points, a cubic spline interpolation and a moving average filtering are then adopted to obtain the virtual FHR baseline. 


\subsection{Fetal movement recognition from actography}

For the fetal movement tracing acquired by actography, Ostu method [17,18] is used to set a threshold automatically to distinguish the fetal movement parts from background noises. The parts above threshold are considered as fetal movement, and the rest are background noises.

To be specific, $T$ is a supposed threshold in Ostu method. By comparing the absolute value of the signals with $T, f_{0}$ is denoted as the ratio of the signals(less than or equal to $T$ )to the whole signals, and the average of these signals is denoted as $u_{0}$, while $f_{1}$ is denoted as the ratio of the signals(greater than $T$ ) to the whole signals, with the average as $u_{1}$. So the average of the whole signals is

$$
u=f_{0} u_{0}+f_{1} u_{1}
$$

So the inter-class variance $g$ of the signals is

$$
g=f_{0}\left(u_{0}-u\right)^{2}+f_{1}\left(u_{1}-u\right)^{2}=f_{0} f_{1}\left(u_{0}-u_{1}\right)^{2}
$$

When $g$ is the greatest, indicating the greatest difference between the two separated parts of signals, the corresponding threshold $T$ is the best threshold. The signals above the best threshold $T$ are considered as fetal movement in this study.

\subsection{Fetal movement extraction from TOCO signals}

Different from fetal movement signals from actography, fetal movement signals involved in TOCO signals mainly composed of uterine contraction signals are weak and imperceptible. Therefore, as a solution, multi-resolution wavelet decomposition is employed to extract fetal movement signals from the embedded background noises. The gist of this method is that the original TOCO signal can be decomposed into the detailed signals $W_{f}\left(2^{j}\right)(0<j \leq J)$ and the signal in the last scale $S_{f}\left(2^{J}\right)$, while the original signal can be recovered through the reverse wavelet transforms with the noise-like detailed signals being removed and useful parts containing fetal movement information being remained.

In this study, the db4 wavelet was adopted and the TOCO signal was decomposed into 8 scales. Based on the experiments and several previous literature $[19,20]$, it is hypothesized that the detailed signal $W_{f}\left(2^{1}\right)$ was associated with the maternal respiration that might give rise to high-frequency interferences, while the $W_{f}\left(2^{4}\right) \sim W_{f}\left(2^{8}\right)$ with the low-frequency interferences included uterine contraction or baseline wandering. Hence, by zeroing these scales of detailed signals, the signals with a higher signal-to-noise ratio (SNR) which predominantly contain the information of fetal movement are reconstructed. 
In order to extract the fetal movement information, Ostu method is also applied to the automatic threshold recognition of the reconstructed signals obtained above, which is the same as the process of automatic fetal movement detection from actography.

\section{Results and discussion}

As shown in Figure 1, for the regular antenatal FHR tracing, Alg1 and Alg2 perform equally well, but the latter is undoubtedly a better choice for the irregular tracing.

As shown in Figure 2, taking the fetal movement felt by the expert as standard, the fetal movement information obtained from actography is more abundant and accurate than that from tocography. Generally speaking, in most cases, actography method outperforms the tocography method.

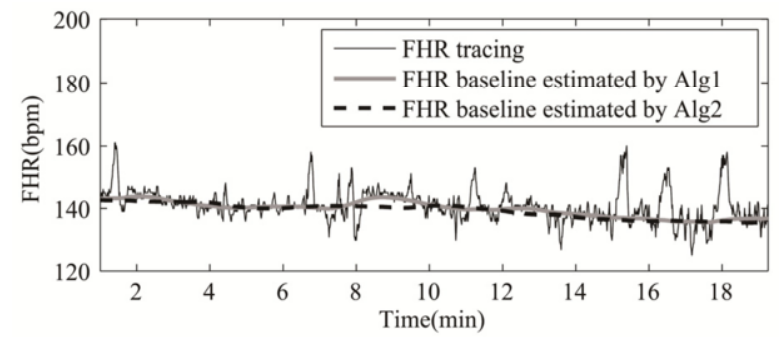

(a)

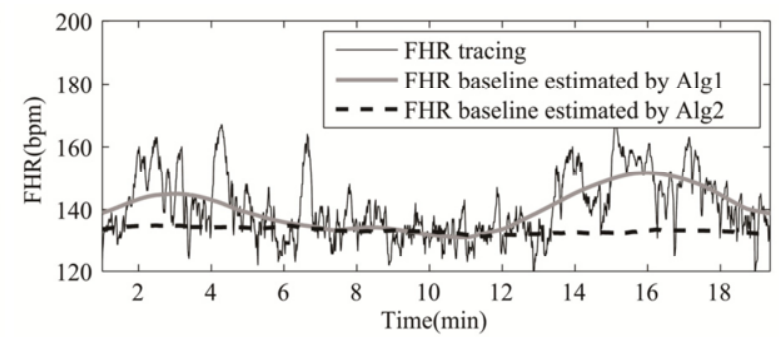

(b)

Fig. 1. The FHR baselines estimated by two algorithms for (a) a regular antenatal FHR tracing with sparsely distributed accelerations, and (b) a typical antenatal FHR tracing with long and frequent accelerations.

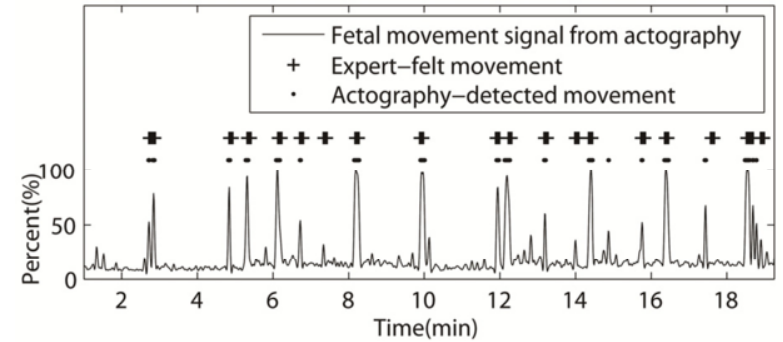

(a)

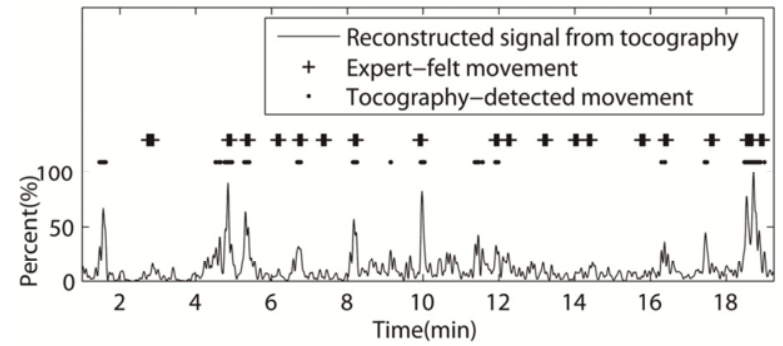

(b)

Fig. 2. The results of automatic fetal movement detections from (a) actography and (b) tocography.

Table 1

The Sensitivity and PPV of four automatic fetal movement detecting methods against the gold standard of 52 cases of antenatal fetal monitoring output

\begin{tabular}{lllll}
\hline Method & Actography & Tocography & Combined method 1 & Combined method 2 \\
\hline sensitivity & 0.7670 & 0.3807 & 0.8205 & 0.3087 \\
PPV & 0.6052 & 0.5000 & 0.5373 & 0.7129 \\
\hline
\end{tabular}




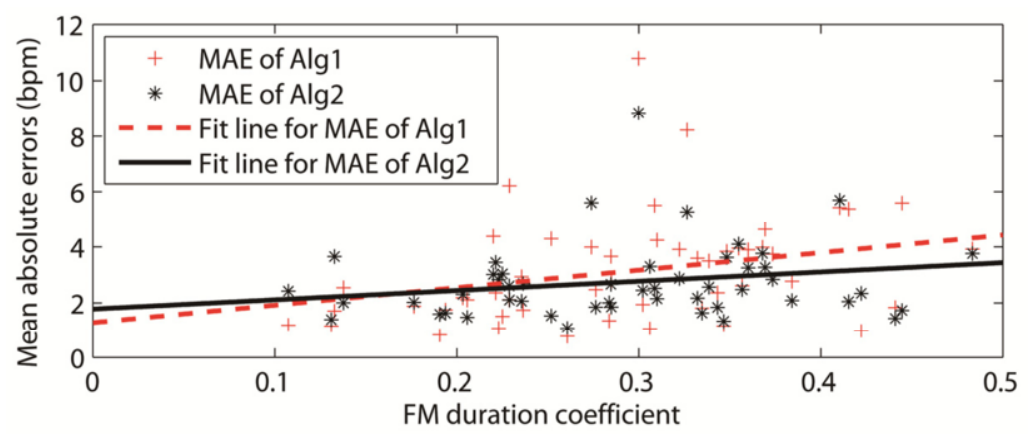

Fig. 3. Mean absolute errors between estimated basal FHR values and the gold standard.

To further assess the accuracy of automatic fetal movement acquired from actography and tocography, the automatic fetal movement is regarded to be accurate when it appears within the $-2.5 \mathrm{~s} \sim 2.5 \mathrm{~s}$ interval of the fetal movement time point detected by the expert. The sensitivity and positive predictive value (PPV) of all the automatic fetal movement detecting methods, including actography, tocography and two different combinations of these two methods, are calculated. Sensitivity is the ratio of accurate automatic fetal movement to the total fetal movement identified by the expert, while PPV is calculated as the ratio of accurate fetal movement to the total fetal movements detected by the algorithms. From Table 1, it is evident that combined method 1, of which the result is the union of results of actography and tocography, has the highest sensitivity, indicating that it identifies more accurate fetal movement. Whereas, combined method 2 shares the highest PPV, whose result is the intersection of results of actography and tocography, showing that the automatic fetal movement identified by this method is most likely to be accurate. Regarding actography and tocography, actography is generally superior to tocography. However, some fetal movement signals are identified by tocography rather than by actography, so tocography should be used in conjunction with actography. Overall, combined method 1 is recommended because of its highest sensitivity and acceptable PPV.

In order to evaluate the baseline estimation results, the expert was asked to give a specific value as the basal FHR value for each of the 52 cases, which is the multiple of $5 \mathrm{bpm}$. Then two baseline algorithms were used to estimate the baselines. For each case, the mean absolute errors (MAE) between the basal FHR value given by the expert and those computed by two algorithms were calculated. The results are shown in Figure 3, in which the scattered points represent the MAEs of estimated basal FHR values from both algorithms with specific fetal movement duration coefficients indicating the frequencies and duration of fetal movement, which is the temporal ratio of recognized fetal movement parts obtained by combined method 1 to the whole fetal movement tracing. Two linear curves are obtained by the least squares method, which fit these points corresponding to two algorithms respectively. The results suggest that $\mathrm{Alg} 2$ may outperform $\mathrm{Alg} 1$ with a greater fetal movement duration coefficient, but the errors of Alg1 could be acceptable when the duration coefficients are within a lower boundary, for example, 0.2 in this study.

\section{Conclusion and future work}

The approaches to extract the fetal movement from actography and tocography have been proposed in this study. It is proven that the combined method which is the union of actography and tocography is valid and feasible. On this basis, a novel approach for FHR baseline estimation has been tested by 
utilizing the information of fetal movement. In essence, this approach is a combination of two baseline estimation algorithms, including EMD-KNN method and a linear baseline estimation method. According to the experimental results, it is concluded that the linear baseline estimation method is recommended when fetal movement duration coefficient is less than 0.2 and the EMD-KNN method is better with a fetal movement duration coefficient over 0.2.

In the future, there will be two directions to further this research: 1. to improve the combinational method continuously by involving more existing baseline estimation algorithms as the material of the resulted method or using more or better parameters as the classification indicator; 2 . to develop a new baseline estimation algorithm by consulting the relationship between FHR and fetal movement.

\section{Acknowledgement}

We appreciated Dr. Ruyi Qin, a specialist obstetrician from the People's Hospital of Baiyun District, Guangzhou, for her help. This work was supported by the Science and Technology Planning Project of Guangdong Province (No. 2012B091000053) and Guangzhou (No. 201207YH017).

\section{References}

[1] G.S. Dawes, C.R. Houghton and C.W. Redman, Baseline in human fetal heart-rate records, BJOG: An International Journal of Obstetrics \& Gynaecology 89 (1982), 270-275.

[2] R. Mantel, H.P. van Geijn, F.J. Caron, J.M. Swartjes, E.E. van Woerden and H.W. Jonsma, Computer analysis of antepartum fetal heart rate: 1, Baseline Determination International Journal of Biomedical Computing 25 (1990), $261-272$.

[3] D. Ayres-de-Campos, J. Bernardes, A. Garrido, J. Marques-de-Sá and L. Pereira-Leite, SisPorto 2.0: A program for automated analysis of cardiotocograms, Journal of Maternal-Fetal \& Neonatal Medicine 9 (2000), 311-318.

[4] L. Jimenez, R. Gonzalez, M. Gaitan, S. Carrasco and C. Vargas, Computerized algorithm for baseline estimation of fetal heart rate, Proceeding of: Computers in Cardiology, 2002, 447-580.

[5] T. Kupka, J. Wrobel, J. Jezewski and A. Gacek, Evaluation of fetal heart rate baseline estimation method using testing signals based on a statistical model, 28th Annual International Conference of the IEEE Engineering in Medicine and Biology Society, 2006, 3728-3731.

[6] B.N. Krupa, M.A.M. Ali and E. Zahedi, Computerized fetal heart rate baseline estimation based on number and continuity of occurrences, i 4th Kuala Lumpur International Conference on Biomedical Engineering IFMBE Proceedings 21 (2008), 162-165.

[7] S. Nidhal, M.A.M. Ali and H. Najah, A novel cardiotocography fetal heart rate baseline estimation algorithm, Scientific Research and Essays 5 (2010), 4002-4010.

[8] S. Andersson, Acceleration and deceleration detection and baseline estimation, M.Sc. Dissertation, Chalmers University of Technology, 2011.

[9] Y. Lu, S. Wei and X. Liu, Nonlinear FHR baseline estimation using empirical mode decomposition and Kohonen neural network, Proceedings of Biomedical Circuits and Systems Conference, 2012, 368-371.

[10] J.A. Dipietro, D.M. Hodgson, K.A. Costigan, S.C. Hilton and T.R. Johnson, Development of fetal movement-fetal heart rate coupling from 20 weeks through term, Early Human Development 44 (1996), 139-151.

[11] C.L. Lowery, W.A. Russell, P.J. Baggot, J.D. Wilson, R.C. Walls, L.S. Bentz and P. Murphy, Time quantified detection of fetal movements using a new fetal movement algorithm, American Journal of Perinatology 14 (1997), 7-12.

[12] L.M. Jansson, J.A. DiPietro, M. Velez, A. Elko, E. Williams, L. Milio, K. O'Grady and H.E. Jones, Fetal neurobehavioral effects of exposure to methadone or buprenorphine, Neurotoxicology and Teratology 33 (2011), 240-243.

[13] R.B. Govindan, S. Vairavan, U.D. Ulusar, J.D. Wilson, S.S. Mckelvey, H. Preissl and H. Eswaran, A novel approach to track fetal movement using multi-sensor magnetocardiographic recordings, Annals of Biomedical Engineering 39 (2011), 964-972.

[14] K. Maeda, M. Tatsumura, K. Nakajima, T. Ida, N. Nagata and Y. Minagawa, The ultrasonic Doppler fetal actocardiogram and its computer processing, Journal of Perinatal Medicine 16 (1988), 327-331. 
[15] W. Schmidt, I. Cseh, K. Hara and F. Kubli, Maternal perception, tocodynamometric findings and real-time ultrasound assessment of total fetal activity, International Journal of Gynaecology \& Obstetrics 22 (1984), 85-90.

[16] V.S. Chourasia and A.K. Tiwari, Design methodology of a new wavelet basis function for fetal phonocardiographic signals, The Scientific World Journal 2013 (2013), 1-12.

[17] N. Otsu, A threshold selection method from gray-level histograms, Journal of electronic imaging 13 (1979), 146-145.

[18] P. Mukherjee, G. Swamy, M. Gupta, U. Patil and K.B. Krishnan, Automatic detection and measurement of femur length from fetal ultrasonography, International Society for Optics and Photonics 7629 (2010), 762909-1-762909-9 .

[19] Y. Xuan, Y. Zhang, Y. Lv, X. Zhao, Z. Yang and P. Yang, Fetal movement signal recognition based on wavelet transform and BP neural network, Journal of Data Acquisition \& Processing 16 (2001), 295-298.

[20] V. Chudáček, J. Spilka, P. Janků, M. Koucký, L. Lhotská and M Huptych, Automatic evaluation of intrapartum fetal heart rate recordings: A comprehensive analysis of useful features, Physiological measurement 32 (2011), 1347-1360. 